\title{
Superconductivity on the Border of Weak Itinerant Ferromagnetism in UCoGe
}

\author{
N. T. Huy, ${ }^{1}$ A. Gasparini, ${ }^{1}$ D. E. de Nijs,${ }^{1}$ Y. Huang, ${ }^{1}$ J. C. P. Klaasse, ${ }^{1}$ T. Gortenmulder,${ }^{1}$ A. de Visser, ${ }^{1, *}$ A. Hamann, ${ }^{2}$ \\ T. Görlach, ${ }^{2}$ and H. v. Löhneysen ${ }^{2,3}$ \\ ${ }^{1}$ Van der Waals-Zeeman Institute, University of Amsterdam, Valckenierstraat 65, 1018 XE Amsterdam, The Netherlands \\ ${ }^{2}$ Physikalisches Institut, Universität Karlsruhe, D-76128 Karlsruhe, Germany \\ ${ }^{3}$ Forschungszentrum Karlsruhe, Institut für Festkörperphysik, D-76021 Karlsruhe, Germany
}

(Received 28 April 2007; published 10 August 2007)

\begin{abstract}
We report the coexistence of ferromagnetic order and superconductivity in UCoGe at ambient pressure. Magnetization measurements show that UCoGe is a weak ferromagnet with a Curie temperature $T_{C}=$ $3 \mathrm{~K}$ and a small ordered moment $m_{0}=0.03 \mu_{B}$. Superconductivity is observed with a resistive transition temperature $T_{s}=0.8 \mathrm{~K}$ for the best sample. Thermal-expansion and specific-heat measurements provide solid evidence for bulk magnetism and superconductivity. The proximity to a ferromagnetic instability, the defect sensitivity of $T_{s}$, and the absence of Pauli limiting, suggest triplet superconductivity mediated by critical ferromagnetic fluctuations.
\end{abstract}

DOI: 10.1103/PhysRevLett.99.067006

PACS numbers: 74.70.Tx, 74.20.Mn, 75.30.Kz

In the standard theory for superconductivity (SC) due to Bardeen, Schrieffer, and Cooper ferromagnetic (FM) order impedes the pairing of electrons in singlet states [1]. It has been argued, however, that on the border line of ferromagnetism, critical magnetic fluctuations could mediate SC by pairing the electrons in triplet states [2]. The discovery several years ago of SC in the metallic ferromagnets $\mathrm{UGe}_{2}$ (at high pressure) [3], URhGe [4], and possibly UIr (at high pressure) [5], has put this idea on firm footing. However, later work provided evidence for a more intricate scenario in which $\mathrm{SC}$ in $\mathrm{UGe}_{2}$ and URhGe is driven by a magnetic transition between two polarized phases [6-8] rather than by critical fluctuations associated with the zero temperature transition from a paramagnetic to a FM phase. Here we report a novel ambient-pressure FM superconductor UCoGe. Since SC occurs right on the border line of FM order, UCoGe may present the first example of SC stimulated by critical fluctuations associated with a FM quantum critical point (QCP).

UCoGe belongs to the family of intermetallic UTX compounds, with $T$ a transition metal and $X$ is $\mathrm{Si}$ or Ge, that was first manufactured by Troć and Tran [9]. UCoGe crystallizes in the orthorhombic TiNiSi structure (space group $\left.P_{n m a}\right)[10,11]$, just like URhGe. From magnetization, resistivity $(T \geq 4.2 \mathrm{~K})[9,10]$ and specific-heat measurements ( $T \geq 1.2 \mathrm{~K})$ [12] it was concluded that UCoGe has a paramagnetic ground state. This provided the motivation to alloy $\mathrm{URhGe}$ (Curie temperature $T_{C}=9.5 \mathrm{~K}$ ) with $\mathrm{Co}$ in a search for a FM QCP in the series $\mathrm{URh}_{1-x} \mathrm{Co}_{x} \mathrm{Ge}(x \leq 0.9)$ [13]. Magnetization data showed that $T_{C}$ upon doping first increases, has a broad maximum near $x=0.6\left(T_{C}^{\max }=20 \mathrm{~K}\right)$ and then rapidly drops to $8 \mathrm{~K}$ for $x=0.9$ [13]. This hinted at a FM QCP for $x \lessgtr 1$.0. In this Letter we show that the end $(x=1.0)$ compound $\mathrm{UCoGe}$ is in fact a weak itinerant ferromagnet. Moreover, metallic ferromagnetism coexists with SC below $0.8 \mathrm{~K}$ at ambient pressure.
Polycrystalline UCoGe samples were prepared with nominal compositions $\mathrm{U}_{1.02} \mathrm{CoGe}$ (sample 2) and $\mathrm{U}_{1.02} \mathrm{Co}_{1.02} \mathrm{Ge}$ (sample 3) by arc melting the constituents (natural U 99.9\%, Co 99.9\%, and Ge 99.999\%) under a high-purity argon atmosphere in a water-cooled copper crucible. The as-cast samples were annealed for 10 days at $850^{\circ} \mathrm{C}$. Samples for the different experiments were cut by spark erosion, after which the defected surface was removed by polishing. Powder x-ray diffraction patterns at $T=300 \mathrm{~K}$ confirmed the TiNiSi structure. The lattice constants extracted are $a=6.845 \AA, b=4.206 \AA$, and $c=7.222 \AA$, in agreement with literature [11]. The phase homogeneity of the annealed samples was investigated by electron microprobe analysis. The matrix has the 1:1:1 composition and all samples contained a small amount (2\%) of impurity phases.

The dc magnetization was measured for temperatures $T \geq 2 \mathrm{~K}$ and magnetic fields $B \leq 5 \mathrm{~T}$ in a SQUID magnetometer. The demagnetizing factor of our samples is small $(N \approx 0.08)$ and corrections due to the demagnetizing field were neglected. Four-point low-frequency acresistivity and ac-susceptibility data were obtained using a phase-sensitive bridge in the range $T=0.02-8 \mathrm{~K}$. The specific heat was measured using a semiadiabatic method employing a mechanical heat switch on a sample with mass $3 \mathrm{~g}$ for $T=0.5-10 \mathrm{~K}$ and with a weak thermal link on a sample with mass $0.1 \mathrm{~g}$ for $T=0.1-1.0 \mathrm{~K}$. Thermalexpansion data were collected using a capacitance dilatometer for $T=0.23-8 \mathrm{~K}$.

In Fig. 1(a) we show $M$ as a function of $T$ (obtained after field cooling). The inflection point in $M(T)$ at $3 \mathrm{~K}$ signals a FM transition with an unusually small ordered moment $m_{0}$. At the lowest temperature $(2 \mathrm{~K})$ the transition is not complete yet, but from the curvature of $M(T)$ the size of $m_{0}$ is estimated to $0.03 \mu_{B}$. FM order is further corroborated by the hysteresis loop in $M(B)$ at $2 \mathrm{~K}$ with a small coercive field of $0.3 \mathrm{mT}$ [see left inset of Fig. 1(a)]. In the right-hand 


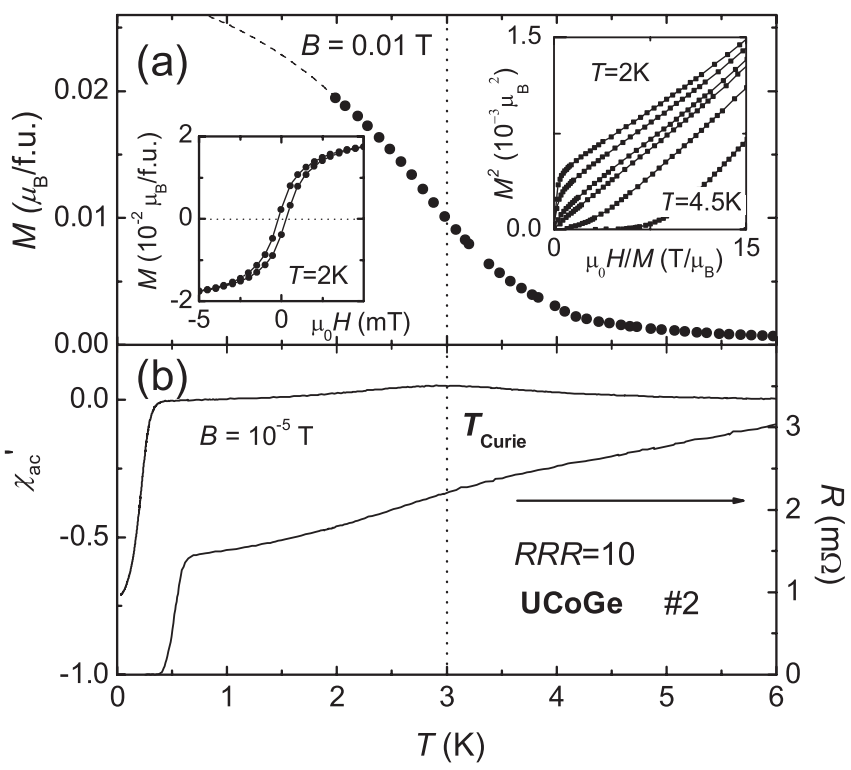

FIG. 1. Magnetic and SC properties of UCoGe sample 2. (a) Magnetization $M$ as a function of $T$ in a field $B$ of $0.01 \mathrm{~T}$. The dashed line extrapolates to $m_{0} \approx 0.03 \mu_{B}$ for $T \rightarrow 0$. The Curie temperature $T_{C}=3 \mathrm{~K}$ is marked by the dotted vertical line. Left inset: Hysteresis loop $M(B)$ at $T=2 \mathrm{~K}$ with coercive field of $0.3 \mathrm{mT}$. Right inset: Arrott plot of magnetization isotherms at $T=2.0,2.4,2.8,3.0,3.5$, and $4.5 \mathrm{~K}$ (from top to bottom). (b) ac susceptibility $\chi_{\text {ac }}^{\prime}$ (left axis) (in $B=10^{-5} \mathrm{~T}$ ), and resistance $R$ (right axis) as a function of $T$. The maximum in $\chi_{\mathrm{ac}}^{\prime}$ and the broad hump in $R$ locate $T_{C}$. SC for sample 2 is found below $0.61 \mathrm{~K}$ in $R(T)$ and below $0.38 \mathrm{~K}$ in $\chi_{\mathrm{ac}}^{\prime}(T)$.

inset of Fig. 1 we show $M$ measured at fixed $T$ between 2 and $4.5 \mathrm{~K}$ in an Arrott plot (i.e., $M^{2}$ versus $\mu_{0} H / M$ ). The isotherm that intersects the origin determines the Curie temperature $T_{C}$. We extract $T_{C}=3 \mathrm{~K}$, in agreement with the $M(T)$ data. The FM transition at $3 \mathrm{~K}$ shows up as a broad peak in the ac susceptibility $\chi_{\mathrm{ac}}^{\prime}(T)[$ Fig. 1(b)] and a hump in the resistance $R(T)$ [Fig. 1(b)]. The magnetic transition is a robust property, as $M(T), \chi_{\mathrm{ac}}^{\prime}(T)$, and $R(T)$ data taken on samples prepared from different batches almost coincide. The small ratio of $m_{0}$ to the effective moment ( $p_{\text {eff }}=1.7 \mu_{B}$ [9]) shows UCoGe is a weak itinerant ferromagnet $[14,15]$.

In Fig. 2 we show the specific heat $c(T)$ and the linear thermal-expansion coefficient, $\quad \alpha(T)=L^{-1} d L / d T$, around the magnetic transition. The transition width is large $\left(\Delta T_{C} \sim 1 \mathrm{~K}\right)$. The relative change $\Delta\left(c / T_{C}\right) /\left(c / T_{C}\right)$ assuming an ideal transition [see dashed line in Fig. 2(a)] is only $25 \%$ and the magnetic entropy associated with the transition is small $(0.3 \%$ of $R \ln 2)$ as expected for a weak itinerant ferromagnet [14] with a small ordered moment. The linear term in the electronic specific heat $\gamma$ amounts to $0.057 \mathrm{~J} / \mathrm{mol} \mathrm{K}^{2}$, which indicates $\mathrm{UCoGe}$ is a correlated metal, but the electron interactions are relatively weak. In $\alpha(T)$ the magnetic transition appears as a large negative contribution. The size of the idealized sharp step $\Delta \alpha$ is $-1.1 \times 10^{-6} \mathrm{~K}^{-1}$ at $T_{C}=3 \mathrm{~K}$ [see dashed line in

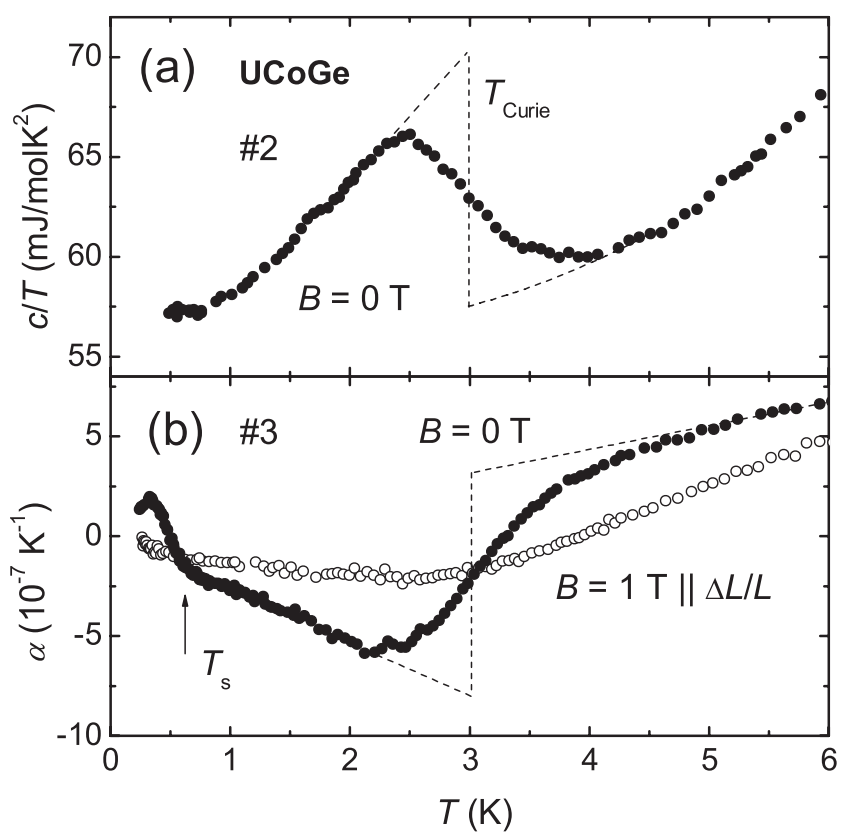

FIG. 2. (a) Specific heat divided by temperature $c / T$ versus $T$ in zero magnetic field for sample 2 . The idealized transition (dashed line) has a step size $\Delta(c / T)=0.014 \mathrm{~J} / \mathrm{mol} \mathrm{K}^{2}$ at $T_{C}=$ $3 \mathrm{~K}$. Bulk SC for sample 2 sets in at $0.38 \mathrm{~K}$ (measured by $\chi_{\mathrm{ac}}^{\prime}$ ), but the $c(T)$ data extend down to $0.5 \mathrm{~K}$ only. (b) Thermalexpansion coefficient $\alpha(T)$ for sample 3. The large negative contribution below $\sim 5 \mathrm{~K}$ is due to FM order. The dashed line gives the idealized transition in $\alpha(T)$ with $\Delta \alpha=-1.1 \times$ $10^{-6} \mathrm{~K}^{-1}$. The total relative length change $\Delta L / L=$ $[L(0.23 \mathrm{~K})-L(T)] / L$ associated with magnetic order is obtained by integrating $\alpha_{\text {mag }}(T)$ (i.e., the difference between the experimental data and the linear term $\alpha=a T$ with $a=1.1 \times$ $10^{-7} \mathrm{~K}^{-2}$ expected in the absence of FM order) and amounts to $+1.9 \times 10^{-6}$. The peak below $\sim 0.6 \mathrm{~K}$ is the thermodynamic signature of the SC transition. In a field of $1 \mathrm{~T}$, applied along the dilatation direction $\Delta L / L$, the magnetic transition is smeared and SC is not resolved.

Fig. 2(b)] and presents a relative change $\Delta \alpha / \alpha$ of $\approx 3.3$. This shows the magnetic transition is a bulk phenomenon.

Below $1 \mathrm{~K}$ UCoGe becomes superconducting as seen by a transition to zero in the resistance $R(T)$ and a large diamagnetic signal in $\chi_{\mathrm{ac}}^{\prime}(T)$; see Figs. 1(b) and 3(a). Unlike the magnetic properties, the SC properties depend sensitively on the quality of the samples as measured by the residual resistance ratio $R R R=R(300 \mathrm{~K}) / R(1 \mathrm{~K})$. For samples $2(R R R=10)$ and $3(R R R=25) \mathrm{SC}$ is found with resistive onset temperatures of $0.61 \mathrm{~K}$ [Fig. 1(b)] and $0.82 \mathrm{~K}$ [Fig. 3(a)], respectively. In these polycrystalline samples the SC transition is relatively broad $\left(\Delta T_{s} \approx\right.$ $0.15 \mathrm{~K})$. The in-phase component of the ac susceptibility $\chi_{\mathrm{ac}}^{\prime}$ starts to drop when the resistive transition is complete. The drop is accompanied by a small dissipative peak in the out-of-phase signal $\chi_{\mathrm{ac}}^{\prime \prime}$ (not plotted). At the lowest $T$ the diamagnetic screening reaches a value of $60 \%-70 \%$ of the ideal screening value $\chi_{M}=-1 /(1-N)$. This indicates $\mathrm{UCoGe}$ is a type II SC which is always in the mixed phase. 

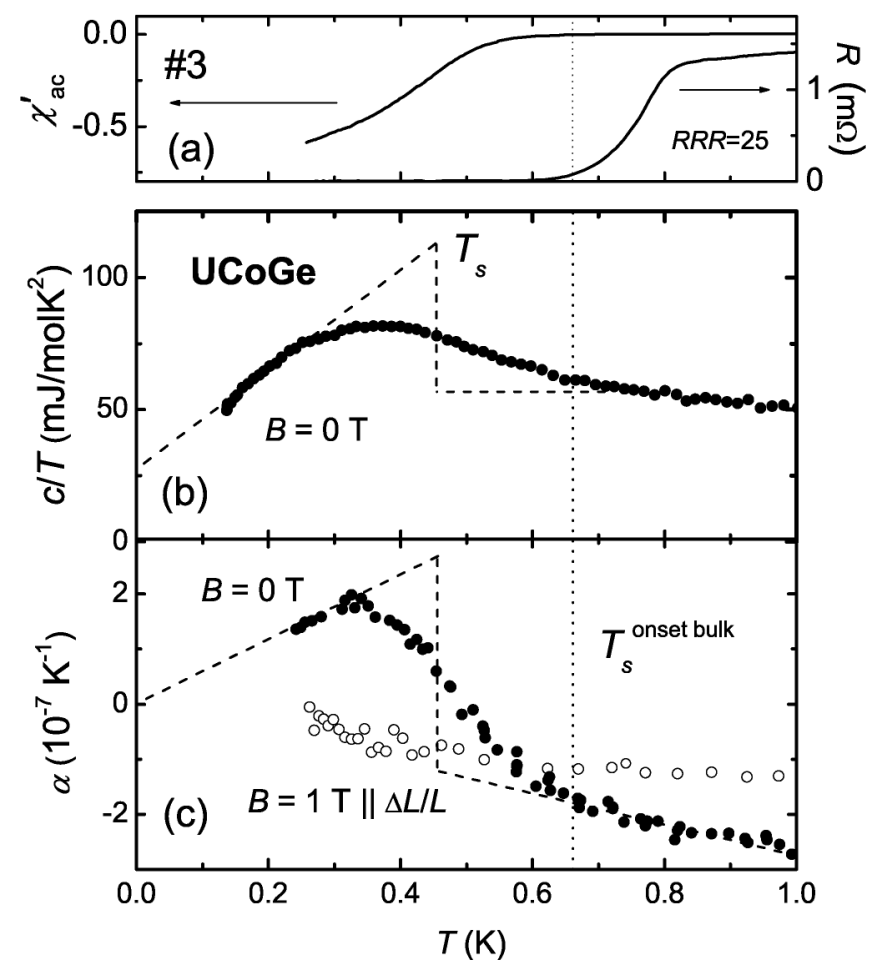

FIG. 3. Superconducting properties of UCoGe sample 3. (a) ac susceptibility $\chi_{\mathrm{ac}}^{\prime}$ (left axis) in $B=10^{-5} \mathrm{~T}$ and resistance $R$ (right axis). (b) Specific heat divided by temperature $c / T$ as a function of $T$. Bulk SC occurs below $T_{s}^{\text {onset }} \approx 0.66 \mathrm{~K}$ (dotted vertical line). Dashed line: Idealized $\mathrm{SC}$ transition using an equal entropy construction with a finite $\gamma$ value in the SC state of $0.028 \mathrm{~J} / \mathrm{mol} \mathrm{K}^{2}$. (c) Coefficient of linear thermal expansion $\alpha(T)$. Bulk SC is observed below $T_{s}^{\text {onset }} \approx 0.66 \mathrm{~K}$. Dashed line: Idealized sharp transition with $T_{s}=0.45 \mathrm{~K}$. For $B=1 \mathrm{~T}$ applied along the dilatation direction $\Delta L / L$ the $\mathrm{SC}$ transition is no longer resolved.

A similar observation [4] with a comparable screening fraction was made for URhGe. Because of the intrinsic FM moments the local field is nonzero and the magnitude of $\chi_{\mathrm{ac}}^{\prime}$ is reduced.

Proof for bulk SC is obtained by specific-heat [Fig. 3(b)] and thermal-expansion measurements [Fig. 3(c)]. The specific heat plotted as $c / T$ versus $T$ shows a broad transition with $T_{s}^{\text {onset }} \approx 0.66 \mathrm{~K}$, which is almost equal to the temperature at which the resistance becomes zero. A rough estimate for the step size of the idealized transition [dashed line in Fig. 3(b)] in the specific heat (at $T_{s} \approx 0.45 \mathrm{~K}$ ) is $\Delta\left(c / T_{s}\right) / \gamma \approx 1.0$, which is smaller than for a conventional $\mathrm{SC}$ (the BCS value is 1.43) but comparable to the value [4] for URhGe. In the thermal expansion an equivalent broad SC transition is observed. Upon entering the SC state $\alpha(T)$ shows a steady increase. We estimate the step size $\Delta \alpha \approx$ $3.8 \times 10^{-7} \mathrm{~K}^{-1}$, assuming an ideal sharp transition [see dashed line in Fig. 3(c)] at $T_{s}=0.45 \mathrm{~K}$. This step size is comparable to the ones (with opposite sign) extracted from thermal-expansion measurements on the heavy-fermion superconductors $\mathrm{URu}_{2} \mathrm{Si}_{2}$ [16] and $\mathrm{UPt}_{3}$ [17,18]. In a magnetic field of $1 \mathrm{~T} \mathrm{SC}$ is suppressed and the thermodynamic signature of the transition is no longer resolved [see Fig. 3(c)]. The $\alpha(T)$ data also show that magnetism and SC coexist. The total relative length change associated with SC, obtained by integrating $\alpha_{\mathrm{sc}}(T)$ after correcting for the normal-state linear contribution $\alpha=a T$ with $a=-2.7 \times$ $10^{-7} \mathrm{~K}^{-2}$ [see dashed line for $0.45 \mathrm{~K} \leq T \leq 1 \mathrm{~K}$ in Fig. 3(c)], amounts to $\Delta L / L=-0.1 \times 10^{-6}$ and is small compared to the length change $\Delta L / L=+1.9 \times 10^{-6}$ due to magnetic ordering (see caption of Fig. 3). Thus magnetism is not expelled below $T_{s}$ and coexists with SC.

In Fig. 4 we show the upper critical field $B_{c 2}(T)$ for samples 2 and 3. The curvature (or tail) of $B_{c 2}$ is attributed to sample inhomogeneities. The quasilinear behavior of $B_{c 2}(T)$ at high fields extrapolates to SC transitions in zero field at 0.30 and $0.60 \mathrm{~K}$. These values are close to $T_{s}^{\text {onset }}$ for bulk SC. From the slope $d B_{c 2} / d T$ and the values of $\gamma$ and the residual resistivity $\rho_{0}$, we can make a crude estimate [19] for the coherence length $(\xi)$ and the mean free path $(\ell)$. For sample $3 d B_{c 2} / d T=-5.2 \mathrm{~T} / \mathrm{K}$ and $\rho_{0}=$ $12 \mu \Omega \mathrm{cm}$, and we calculate $\xi \approx 150 \AA$ and $\ell \approx 500 \AA$. This indicates sample 3 satisfies the clean-limit condition $(\ell>\xi)$, a prerequisite for unconventional SC [20]. For the less pure sample 2 we find $\xi \approx 200 \AA$ and $\ell \approx 300 \AA$. The value of $B_{c 2}$ at the lowest $T$ exceeds the BCS Pauli paramagnetic limit [19] $\left(B_{c 2}^{\text {Pauli }}=1.8 T_{s} \approx 1 \mathrm{~T}\right.$ for sample 3$)$, which for spin-singlet pairing is only possible in the case of strong spin-orbit scattering. On the other hand, the absence of Pauli limiting is expected for a triplet SC with equalspin pairing state [21].

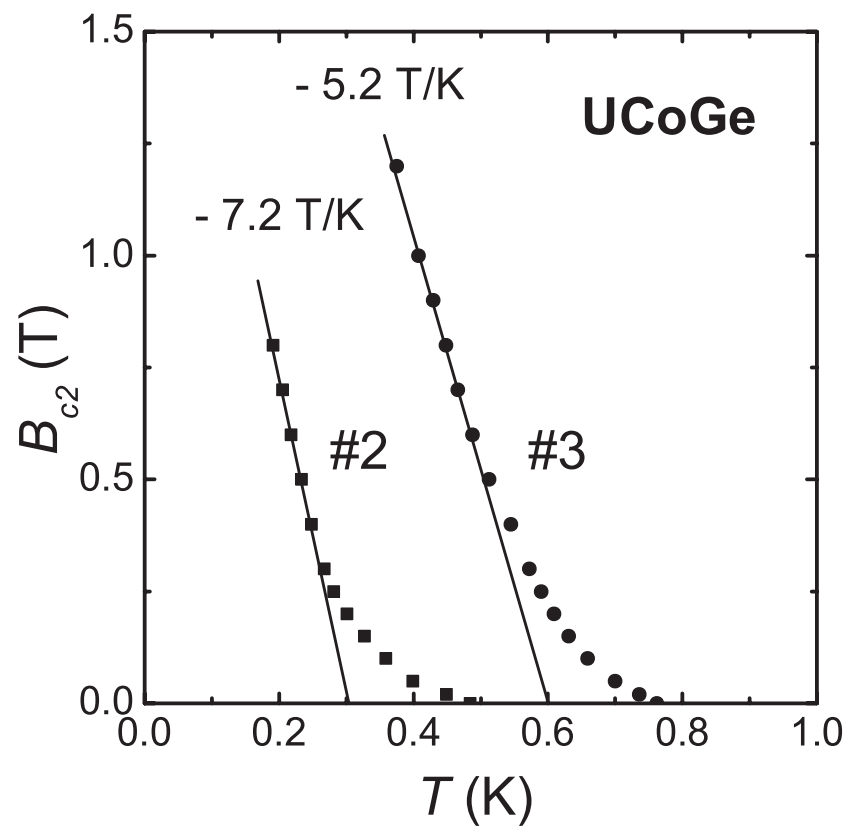

FIG. 4. Upper critical field $B_{c 2}$ determined by the midpoints of the resistive transitions measured in fixed magnetic fields. The solid lines indicate $B_{c 2} / d T=-7.2$ and $-5.2 \mathrm{~T} / \mathrm{K}$ for samples 2 and 3 , respectively, and extrapolate to zero field $T_{s}$ values of 0.30 and $0.60 \mathrm{~K}$. 
The small ordered moment of $0.03 \mu_{B}$ and low Curie temperature locate $\mathrm{UCoGe}$ close to the FM instability (i.e., the limit $T_{C} \rightarrow 0$ ). The proximity to the FM QCP can be further investigated using the Ehrenfest relation for second-order phase transitions $d T_{C} / d p=V_{m} T_{C} \Delta \alpha / \Delta c$ (with the molar volume $V_{m}=3.13 \times 10^{-5} \mathrm{~m}^{3} / \mathrm{mol}$ ). From the estimated step sizes in $\alpha(T)$ and $c(T)$ at $T_{C}$ we calculate $d T_{C} / d p=-0.25 \mathrm{~K} / \mathrm{kbar}$. This shows that the critical pressure $p_{c}$ at which magnetism vanishes is low (an upper bound for $p_{c}$ assuming a linear suppression of $T_{C}$ is $\sim 12$ kbar). In the same way we find that the SC transition temperature increases with pressure at a rate $d T_{s} / d p \approx$ $0.048 \mathrm{~K} / \mathrm{kbar}$. In the scenario of the coexistence of $p$-state $\mathrm{SC}$ and FM [2], the increase of $T_{s}$ with pressure places $\mathrm{UCoGe}$ in the phase diagram on the far side of the SC lobe with respect to the critical point (compare $\mathrm{UGe}_{2}$ at pressures of 10-12 kbar [3]). Accordingly, upon applying pressure, $T_{s}$ is predicted to pass through a maximum before vanishing at the magnetic critical point. The derived pressure dependencies of $T_{C}$ and $T_{s}$ for UCoGe have an opposite sign compared to those for URhGe. In URhGe $T_{C}$ shows a monotonic increase under pressures up to $120 \mathrm{kbar}$ [22] and $T_{s}$ is suppressed with pressure. The positive pressure dependence of $T_{s}$ in UCoGe may explain the large difference in onset temperatures for superconductivity in the transport and bulk properties. Positive stress at the grain boundaries could cause a small volume fraction of the samples to have a larger $T_{s}$.

The occurrence of SC in a FM material is naturally explained [2] by the formation of Cooper pairs with parallel spin. In UCoGe the proximity to the magnetic instability, the defect sensitivity of $T_{s}$, and the absence of Pauli limiting are all in agreement with such a scenario. Within the symmetry classification for orthorhombic itinerant FM spin-triplet superconductors [23] the SC gap is predicted to be anisotropic with point nodes along the magnetic moment direction or line nodes in the plane perpendicular to the moments. The determination of the gap function, however, requires experiments on single crystals. In the case of URhGe, which belongs to the same symmetry class as UCoGe, upper critical field measurements [24] on a single crystal indicate a $p$-wave polar order parameter with a maximum gap parallel to the $a$ axis (the order moment points along the $c$ axis [4]). The difference of a factor of 7 in the size of the ordered moment $m_{0}$ (for URhGe the powder-averaged moment is $m_{0} \approx 0.21 \mu_{B}$ [4]) and the opposite pressure effects on $T_{C}$ and $T_{s}$ seem to indicate that $\mathrm{UCoGe}$ and $\mathrm{URhGe}$ represent two different cases of magnetically mediated SC. Indeed the recent observation of field-induced SC [8] in URhGe was taken as evidence for SC stimulated by a spin rotation in the neighborhood of a quantum phase transition under high magnetic field. In the case of $\mathrm{UGe}_{2}$ the situation is again different as the FM to paramagnetic transition at the critical pressure becomes first order [3]. Moreover, evidence [6,7] is available that $\mathrm{SC}$ is driven by a changing Fermi surface topology associated with a metamagnetic jump in the magnetization.
Consequently, unlike $\mathrm{URhGe}$ and $\mathrm{UGe}_{2}$, $\mathrm{UCoGe}$ may present a genuine case of SC at a FM quantum critical point.

In conclusion, we have demonstrated that $\mathrm{UCoGe}$ is a weak ferromagnet below $T_{C}=3 \mathrm{~K}$ and becomes superconducting upon further cooling with $T_{s}=0.8 \mathrm{~K}$ for the best sample. The sizable discontinuities in the thermodynamic properties at both transition temperatures provide evidence for the bulklike nature of both states. The coexistence of FM and SC is unusual and suggests SC mediated by magnetic interactions rather than by phonons. Since both SC and FM occur at ambient pressure, UCoGe offers a unique opportunity to elucidate the long-standing issue of SC stimulated by critical fluctuations associated with a magnetic quantum critical point.

This work was part of the research program of FOM (Dutch Foundation for Fundamental Research of Matter) and COST Action P16 ECOM. Funding by the Helmholtz Association under No. VH-VI 127 is gratefully acknowledged.

*devisser@science.uva.nl

[1] N. F. Berk and J. R. Schrieffer, Phys. Rev. Lett. 17, 433 (1966).

[2] D. Fay and J. Appel, Phys. Rev. B 22, 3173 (1980).

[3] S. S. Saxena et al., Nature (London) 406, 587 (2000).

[4] D. Aoki et al., Nature (London) 413, 613 (2001).

[5] T. Akazawa et al., J. Phys. Condens. Matter 16, L29 (2004).

[6] C. Pfleiderer and A.D. Huxley, Phys. Rev. Lett. 89, 147005 (2002).

[7] K. G. Sandeman, G. G. Lonzarich, and A. J. Schofield, Phys. Rev. Lett. 90, 167005 (2003).

[8] F. Lévy et al., Science 309, 1343 (2005).

[9] R. Troć and V.H. Tran, J. Magn. Magn. Mater. 73, 389 (1988).

[10] B. Lloret, Ph.D. thesis, University Bordeaux I, 1988.

[11] F. Canepa et al., J. Alloys Compd. 234, 225 (1996).

[12] K. H. J. Buschow et al., J. Appl. Phys. 67, 5215 (1990).

[13] S. Sakarya et al., arXiv:cond-mat/0609557v2 [J. Alloys Comp. (to be published)].

[14] E. P. Wohlfarth, Physica (Amsterdam) 91B, 305 (1977).

[15] T. Moriya, Spin Fluctuations in Itinerant Electron Magnetism (Springer, Berlin, 1985).

[16] N.H. van Dijk et al., Phys. Rev. B 51, 12665 (1995).

[17] N.H. van Dijk et al., J. Low Temp. Phys. 93, 101 (1993).

[18] N. H. van Dijk et al., Phys. Rev. B 48, 1299 (1993).

[19] T. P. Orlando et al., Phys. Rev. B 19, 4545 (1979).

[20] A. J. Millis, S. Sachdev, and C. M. Varma, Phys. Rev. B 37, 4975 (1988).

[21] R. A. Klemm and K. Scharnberg, Physica (Amsterdam) 135B, 53 (1985).

[22] F. Hardy et al., Physica (Amsterdam) 359B, 1111 (2005).

[23] V.P. Mineev and T. Champel, Phys. Rev. B 69, 144521 (2004).

[24] F. Hardy and A. D. Huxley, Phys. Rev. Lett. 94, 247006 (2005). 